\title{
Inequalities of Jensen Type for $h$-Convex Functions on Linear Spaces
}

\author{
Silvestru Sever Dragomir
}

\begin{abstract}
Some inequalities of Jensen type for $h$-convex functions defined on convex subsets in real or complex linear spaces are given. Applications for norm inequalities are provided as well.
\end{abstract}

\section{INTRODUCTION}

We recall here some concepts of convexity that are well known in the literature.

Let $I$ be an interval in $\mathbb{R}$.

Definition 1 ( [38]). We say that $f: I \rightarrow \mathbb{R}$ is a Godunova-Levin function or that $f$ belongs to the class $Q(I)$ if $f$ is non-negative and for all $x, y \in I$ and $t \in(0,1)$ we have

$$
f(t x+(1-t) y) \leq \frac{1}{t} f(x)+\frac{1}{1-t} f(y) .
$$

Some further properties of this class of functions can be found in $[28,29$, $31,44,47,48]$. Among others, its has been noted that non-negative monotone and non-negative convex functions belong to this class of functions.

The above concept can be extended for functions $f: C \subseteq X \rightarrow[0, \infty)$ where $C$ is a convex subset of the real or complex linear space $X$ and the inequality (1.1) is satisfied for any vectors $x, y \in C$ and $t \in(0,1)$. If the function $f: C \subseteq X \rightarrow \mathbb{R}$ is non-negative and convex, then is of GodunovaLevin type.

Definition 2 ( [31]). We say that a function $f: I \rightarrow \mathbb{R}$ belongs to the class $P(I)$ if it is nonnegative and for all $x, y \in I$ and $t \in[0,1]$ we have

$$
f(t x+(1-t) y) \leq f(x)+f(y) .
$$

Obviously $Q(I)$ contains $P(I)$ and for applications it is important to note that also $P(I)$ contain all nonnegative monotone, convex and quasi convex functions, i. e. nonnegative functions satisfying

1991 Mathematics Subject Classification. Primary: 26D15, 25D10.

Key words and phrases. Convex functions, Integral inequalities, $h$-Convex functions. 


$$
f(t x+(1-t) y) \leq \max \{f(x), f(y)\}
$$

for all $x, y \in I$ and $t \in[0,1]$.

For some results on $P$-functions see [31] and [45] while for quasi convex functions, the reader can consult [30].

If $f: C \subseteq X \rightarrow[0, \infty)$, where $C$ is a convex subset of the real or complex linear space $X$, then we say that it is of $P$-type (or quasi-convex) if the inequality (1.2) (or (1.3)) holds true for $x, y \in C$ and $t \in[0,1]$.

Definition 3 ( [7]). Let $s$ be a real number, $s \in(0,1]$. A function $f$ : $[0, \infty) \rightarrow[0, \infty)$ is said to be s-convex (in the second sense) or Breckner $s$-convex if

$$
f(t x+(1-t) y) \leq t^{s} f(x)+(1-t)^{s} f(y)
$$

for all $x, y \in[0, \infty)$ and $t \in[0,1]$.

For some properties of this class of functions see $[1,2,7,8,26,27,39,41,50]$.

The concept of Breckner s-convexity can be similarly extended for functions defined on convex subsets of linear spaces.

It is well known that if $(X,\|\cdot\|)$ is a normed linear space, then the function $f(x)=\|x\|^{p}, p \geq 1$ is convex on $X$.

Utilising the elementary inequality $(a+b)^{s} \leq a^{s}+b^{s}$ that holds for any $a, b \geq 0$ and $s \in(0,1]$, we have for the function $g(x)=\|x\|^{s}$ that

$$
\begin{aligned}
g(t x+(1-t) y) & =\|t x+(1-t) y\|^{s} \leq(t\|x\|+(1-t)\|y\|)^{s} \\
& \leq(t\|x\|)^{s}+[(1-t)\|y\|]^{s} \\
& =t^{s} g(x)+(1-t)^{s} g(y)
\end{aligned}
$$

for any $x, y \in X$ and $t \in[0,1]$, which shows that $g$ is Breckner $s$-convex on $X$.

In order to unify the above concepts for functions of real variable, S. Varošanec introduced the concept of $h$-convex functions as follows.

Assume that $I$ and $J$ are intervals in $\mathbb{R},(0,1) \subseteq J$ and functions $h$ and $f$ are real non-negative functions defined in $J$ and $I$, respectively.

Definition 4 ( [53]). Let $h: J \rightarrow[0, \infty)$ with $h$ not identical to 0 . We say that $f: I \rightarrow[0, \infty)$ is an $h$-convex function if for all $x, y \in I$ we have

$$
f(t x+(1-t) y) \leq h(t) f(x)+h(1-t) f(y)
$$

for all $t \in(0,1)$.

For some results concerning this class of functions see [6, 42, 49, 51-53].

This concept can be extended for functions defined on convex subsets of linear spaces in the same way as above replacing the interval $I$ be the corresponding convex subset $C$ of the linear space $X$.

We can introduce now another class of functions. 
Definition 5. We say that the function $f: C \subseteq X \rightarrow[0, \infty)$ is of $s$ Godunova-Levin type, with $s \in[0,1]$, if

$$
f(t x+(1-t) y) \leq \frac{1}{t^{s}} f(x)+\frac{1}{(1-t)^{s}} f(y),
$$

for all $t \in(0,1)$ and $x, y \in C$.

We observe that for $s=0$ we obtain the class of $P$-functions while for $s=1$ we obtain the class of Godunova-Levin. If we denote by $Q_{s}(C)$ the class of $s$-Godunova-Levin functions defined on $C$, then we obviously have

$$
P(C)=Q_{0}(C) \subseteq Q_{s_{1}}(C) \subseteq Q_{s_{2}}(C) \subseteq Q_{1}(C)=Q(C)
$$

for $0 \leq s_{1} \leq s_{2} \leq 1$.

For different inequalities related to these classes of functions, see $[1-4,6$, 9-37, 40-42, 45-52].

A function $h: J \rightarrow \mathbb{R}$ is said to be supermultiplicative if

$$
h(t s) \geq h(t) h(s) \text { for any } t, s \in J .
$$

If the inequality (1.6) is reversed, then $h$ is said to be submultiplicative. If the equality holds in (1.6) then $h$ is said to be a multiplicative function on $J$.

In [53] it has been noted that if $h:[0, \infty) \rightarrow[0, \infty)$ with $h(t)=(x+c)^{p-1}$, then for $c=0$ the function $h$ is multiplicative. If $c \geq 1$, then for $p \in(0,1)$ the function $h$ is supermultiplicative and for $p>1$ the function is submultiplicative.

We observe that, if $h, g$ are nonnegative and supermultiplicative, the same is their product. In particular, if $h$ is supermultiplicative then its product with a power function $\ell_{r}(t)=t^{r}$ is also supermultiplicative.

The case of $h$-convex function with $h$ supermultiplicative is of interest due to several Jensen type inequalities one can derive.

The following results were obtained in [53] for functions of a real variable. However, with similar proofs they can be extended to $h$-convex function defined on convex subsets in linear spaces.

Theorem 1. Let $h: J \rightarrow[0, \infty)$ be a supermultiplicative function on $J$. If the function $f: C \subseteq X \rightarrow[0, \infty)$ is $h$-convex on the convex subset $C$ of the linear space $X$, then for any $w_{i} \geq 0, i \in\{1, \ldots, n\}, n \geq 2$ with $W_{n}:=\sum_{i=1}^{n} w_{i}>0$ we have

$$
f\left(\frac{1}{W_{n}} \sum_{i=1}^{n} w_{i} x_{i}\right) \leq \sum_{i=1}^{n} h\left(\frac{w_{i}}{W_{n}}\right) f\left(x_{i}\right) .
$$

In particular, we have the unweighted inequality

$$
f\left(\frac{1}{n} \sum_{i=1}^{n} x_{i}\right) \leq h\left(\frac{1}{n}\right) \sum_{i=1}^{n} f\left(x_{i}\right) .
$$


Corollary 1 ( [27]). If the function $f: C \subseteq X \rightarrow[0, \infty)$ is Breckner $s$ convex on the convex subset $C$ of the linear space $X$ with $s \in(0,1)$, then for any $x_{i} \in C, w_{i} \geq 0, i \in\{1, \ldots, n\}, n \geq 2$ with $W_{n}:=\sum_{i=1}^{n} w_{i}>0$ we have

$$
f\left(\frac{1}{W_{n}} \sum_{i=1}^{n} w_{i} x_{i}\right) \leq \frac{1}{W_{n}^{s}} \sum_{i=1}^{n} w_{i}^{s} f\left(x_{i}\right) .
$$

If $(X,\|\cdot\|)$ is a normed linear space, then for $s \in(0,1), x_{i} \in X, w_{i} \geq 0$, $i \in\{1, \ldots, n\}, n \geq 2$ with $W_{n}:=\sum_{i=1}^{n} w_{i}>0$ we have the norm inequality

$$
\left\|\sum_{i=1}^{n} w_{i} x_{i}\right\|^{s} \leq \sum_{i=1}^{n} w_{i}^{s}\left\|x_{i}\right\|^{s} .
$$

Corollary 2. If the function $f: C \subseteq X \rightarrow[0, \infty)$ is of s-Godunova-Levin type, with $s \in[0,1]$, on the convex subset $C$ of the linear space $X$, then for any $x_{i} \in C, w_{i}>0, i \in\{1, \ldots, n\}, n \geq 2$ we have

$$
f\left(\frac{1}{W_{n}} \sum_{i=1}^{n} w_{i} x_{i}\right) \leq W_{n}^{s} \sum_{i=1}^{n} \frac{1}{w_{i}^{s}} f\left(x_{i}\right) .
$$

This result generalizes the Jensen type inequality obtained in [44] for $s=1$.

Let $K$ be a finite non-empty set of positive integers. We can define the index set function, see also [53]

$$
J(K):=\sum_{i \in K} h\left(w_{i}\right) f\left(x_{i}\right)-h\left(W_{K}\right) f\left(\frac{1}{W_{K}} \sum_{i \in K} w_{i} x_{i}\right),
$$

where $W_{K}:=\sum_{i \in K} w_{i}>0, x_{i} \in C, i \in K$.

We notice that if $h:[0, \infty) \rightarrow[0, \infty)$ is a supermultiplicative function on $[0, \infty)$ and the function $f: C \subseteq X \rightarrow[0, \infty)$ is $h$-convex on the convex subset $C$ of the linear space $X$, then

$$
J(K) \geq h\left(W_{K}\right)\left[\sum_{i \in K} h\left(\frac{w_{i}}{W_{K}}\right) f\left(x_{i}\right)-f\left(\frac{1}{W_{K}} \sum_{i \in K} w_{i} x_{i}\right)\right] \geq 0 .
$$

Theorem 2. Assume that $h:[0, \infty) \rightarrow[0, \infty)$ is a supermultiplicative function on $[0, \infty)$ and the function $f: C \subseteq X \rightarrow[0, \infty)$ is $h$-convex on the convex subset $C$ of the linear space $X$. Let $M$ and $K$ be finite non-empty sets of positive integers, $w_{i}>0, x_{i} \in C, i \in K \cup M$. Then

$$
J(K \cup M) \geq J(K)+J(M) \geq 0,
$$

i.e., $J$ is a superadditive index set functional.

This results was proved in an equivalent form in [53] for functions of a real variable. The proof is similar for functions defined on convex sets in linear spaces. 
Corollary 3. With the assumptions of Theorem 2 and if we note $M_{k}:=$ $\{1, \ldots, k\}$, then

$$
J\left(M_{n}\right) \geq J\left(M_{n-1}\right) \geq \ldots \geq J\left(M_{2}\right) \geq 0
$$

and

$$
\begin{array}{r}
J\left(M_{n}\right) \geq \max _{1 \leq i<j \leq n}\left\{h\left(w_{i}\right) f\left(x_{i}\right)+h\left(w_{j}\right) f\left(x_{j}\right)\right. \\
\left.-h\left(w_{i}+w_{j}\right) f\left(\frac{w_{i} x_{i}+w_{j} x_{j}}{w_{i}+w_{j}}\right)\right\}
\end{array}
$$

$$
\geq 0
$$

If we consider the functional

$$
J_{s}(K):=\sum_{i \in K} w_{i}^{s}\left\|x_{i}\right\|^{s}-\left\|\sum_{i \in K} w_{i} x_{i}\right\|^{s}
$$

for $s \in(0,1)$, then we have the norm inequalities

$$
\begin{aligned}
\sum_{i=1}^{n} w_{i}^{s}\left\|x_{i}\right\|^{s}-\left\|\sum_{i=1}^{n} w_{i} x_{i}\right\|^{s} & \geq \sum_{i=1}^{n-1} w_{i}^{s}\left\|x_{i}\right\|^{s}-\left\|\sum_{i=1}^{n-1} w_{i} x_{i}\right\|^{s} \\
& \geq \ldots \geq \sum_{i=1}^{2} w_{i}^{s}\left\|x_{i}\right\|^{s}-\left\|\sum_{i=1}^{2} w_{i} x_{i}\right\|^{s} \geq 0
\end{aligned}
$$

and

$$
\begin{aligned}
& \sum_{i=1}^{n} w_{i}^{s}\left\|x_{i}\right\|^{s}-\left\|\sum_{i=1}^{n} w_{i} x_{i}\right\|^{s} \\
& \geq \max _{1 \leq i<j \leq n}\left\{w_{i}^{s}\left\|x_{i}\right\|^{s}+w_{j}^{s}\left\|x_{j}\right\|^{s}-\left\|w_{i} x_{i}+w_{j} x_{j}\right\|^{s}\right\} \geq 0
\end{aligned}
$$

where $w_{i} \geq 0, x_{i} \in X, i \in\{1, \ldots, n\}, n \geq 2$.

\section{More Jensen Type Results}

Let $h(z)=\sum_{n=0}^{\infty} a_{n} z^{n}$ be a power series with complex coefficients and convergent on the open disk $D(0, R) \subset \mathbb{C}, R>0$. We have the following 
examples

$$
\begin{aligned}
& h(z)=\sum_{n=1}^{\infty} \frac{1}{n} z^{n}=\ln \frac{1}{1-z}, \quad z \in D(0,1) \\
& h(z)=\sum_{n=0}^{\infty} \frac{1}{(2 n) !} z^{2 n}=\cosh z, \quad z \in \mathbb{C} ; \\
& h(z)=\sum_{n=0}^{\infty} \frac{1}{(2 n+1) !} z^{2 n+1}=\sinh z, \quad z \in \mathbb{C} ; \\
& h(z)=\sum_{n=0}^{\infty} z^{n}=\frac{1}{1-z}, \quad z \in D(0,1) .
\end{aligned}
$$

Other important examples of functions as power series representations with nonnegative coefficients are:

$$
\begin{aligned}
h(z) & =\sum_{n=0}^{\infty} \frac{1}{n !} z^{n}=\exp (z), \quad z \in \mathbb{C} \\
h(z) & =\sum_{n=1}^{\infty} \frac{1}{2 n-1} z^{2 n-1}=\frac{1}{2} \ln \left(\frac{1+z}{1-z}\right), \quad z \in D(0,1) \\
h(z) & =\sum_{n=0}^{\infty} \frac{\Gamma\left(n+\frac{1}{2}\right)}{\sqrt{\pi}(2 n+1) n !} z^{2 n+1}=\sin ^{-1}(z), \quad z \in D(0,1) \\
h(z) & =\sum_{n=1}^{\infty} \frac{1}{2 n-1} z^{2 n-1}=\tanh ^{-1}(z), \quad z \in D(0,1) \\
h(z) & ={ }_{2} F_{1}(\alpha, \beta, \gamma, z)=\sum_{n=0}^{\infty} \frac{\Gamma(n+\alpha) \Gamma(n+\beta) \Gamma(\gamma)}{n ! \Gamma(\alpha) \Gamma(\beta) \Gamma(n+\gamma)} z^{n}, \alpha, \beta, \gamma>0 \\
z & \in D(0,1) ;
\end{aligned}
$$

where $\Gamma$ is Gamma function.

The following result may provide many examples of supemultiplicative functions.

Theorem 3. Let $h(z)=\sum_{n=0}^{\infty} a_{n} z^{n}$ be a power series with complex coefficients and convergent on the open disk $D(0, R) \subset \mathbb{C}, R>0$. Assume that $0<r<R$ and define $h_{r}:[0,1] \rightarrow[0, \infty), h_{r}(t):=\frac{h(r t)}{h(r)}$. Then $h_{r}$ is supemultiplicative on $[0,1]$.

Proof. We use the Čebyšev inequality for synchronous (the same monotonicity) sequences $\left(c_{i}\right)_{i \in \mathbb{N}},\left(b_{i}\right)_{i \in \mathbb{N}}$ and nonnegative weights $\left(p_{i}\right)_{i \in \mathbb{N}}$ :

$$
\sum_{i=0}^{n} p_{i} \sum_{i=0}^{n} p_{i} c_{i} b_{i} \geq \sum_{i=0}^{n} p_{i} c_{i} \sum_{i=0}^{n} p_{i} b_{i}
$$


for any $n \in \mathbb{N}$.

Let $t, s \in(0,1)$ and define the sequences $c_{i}:=t^{i}, b_{i}:=s^{i}$. These sequences are decreasing and if we apply Čbyšev's inequality for these sequences and the weights $p_{i}:=a_{i} r^{i} \geq 0$ we get

$$
\sum_{i=0}^{n} a_{i} r^{i} \sum_{i=0}^{n} a_{i}(r t s)^{i} \geq \sum_{i=0}^{n} a_{i}(r t)^{i} \sum_{i=0}^{n} a_{i}(r s)^{i}
$$

for any $n \in \mathbb{N}$.

Since the series

$$
\sum_{i=0}^{\infty} a_{i} r^{i}, \quad \sum_{i=0}^{\infty} a_{i}(r t s)^{i}, \quad \sum_{i=0}^{\infty} a_{i}(r t)^{i} \text { and } \sum_{i=0}^{\infty} a_{i}(r s)^{i}
$$

are convergent, then by letting $n \rightarrow \infty$ in (2.4) we get

$$
h(r) h(r t s) \geq h(r t) h(r s)
$$

i.e.

$$
h_{r}(t s) \geq h_{r}(t) h_{r}(s) .
$$

This inequality is also obviously satisfied at the end points of the interval $[0,1]$ and the proof is completed.

Remark 1. Utilising the above theorem, we then conclude that the functions

$$
h_{r}:[0,1] \rightarrow[0, \infty), h_{r}(t):=\frac{1-r}{1-r t}, r \in(0,1)
$$

and

$$
h_{r}:[0,1] \rightarrow[0, \infty), h_{r}(t):=\exp [-r(1-t)], r>0
$$

are supermultiplicative.

We say that the function $f: C \subseteq X \rightarrow[0, \infty)$ is $r$-resolvent convex with $r$ fixed in $(0,1)$, if $f$ is $h$-convex with $h(t)=\frac{1-r}{1-r t}$, i.e.

$$
f(t x+(1-t) y) \leq(1-r)\left[\frac{1}{1-r t} f(x)+\frac{1}{1-r+r t} f(y)\right]
$$

for any $x, y \in C$ and $t \in[0,1]$.

In particular, for $r=\frac{1}{2}$ we have $\frac{1}{2}$-resolvent convex functions defined by the condition

$$
f(t x+(1-t) y) \leq \frac{1}{2-t} f(x)+\frac{1}{1+t} f(y)
$$

for any $t \in[0,1]$ and $x, y \in C$.

Since

$$
t<\frac{1}{2-t}<\frac{1}{t} \text { and } 1-t<\frac{1}{1+t}<\frac{1}{1-t} \text { for } t \in(0,1)
$$

it follows that any nonnegative convex function is $\frac{1}{2}$-resolvent convex which, in its turn, is of Godunova-Levin type. 
We say that the function $f: C \subseteq X \rightarrow[0, \infty)$ is $r$-exponential convex with $r$ fixed in $(0, \infty)$, if $f$ is $h$-convex with $h(t)=\exp [-r(1-t)]$, i.e.

$$
f(t x+(1-t) y) \leq \exp [-r(1-t)] f(x)+\exp (-r t) f(y)
$$

for any $t \in[0,1]$ and $x, y \in C$.

Since

$$
t \leq \exp [-r(1-t)] \text { and } 1-t \leq \exp (-r t) \text { for } t \in[0,1]
$$

it follows that any nonnegative convex function is $r$-exponential convex with $r \in(0, \infty)$.

Corollary 4. Let $h(z)=\sum_{n=0}^{\infty} a_{n} z^{n}$ be a power series with complex coefficients and convergent on the open disk $D(0, R) \subset \mathbb{C}, R>0$. Assume that $0<r<R$ and define $h_{r}:[0,1] \rightarrow[0, \infty), h_{r}(t):=\frac{h(r t)}{h(r)}$. If the function $f: C \subseteq X \rightarrow[0, \infty)$ is $h_{r}$-convex on the convex subset $C$ of the linear space $X$, namely

$$
f(t x+(1-t) y) \leq \frac{1}{h(r)}[h(r t) f(x)+h(r(1-t)) f(y)]
$$

for any $t \in[0,1]$ and $x, y \in C$, then for any $x_{i} \in C, w_{i} \geq 0, i \in\{1, \ldots, n\}$, $n \geq 2$ with $W_{n}:=\sum_{i=1}^{n} w_{i}>0$ we have

$$
f\left(\frac{1}{W_{n}} \sum_{i=1}^{n} w_{i} x_{i}\right) \leq \frac{1}{h(r)} \sum_{i=1}^{n} h\left(r \frac{w_{i}}{W_{n}}\right) f\left(x_{i}\right) .
$$

Remark 2. If the function $f: C \subseteq X \rightarrow[0, \infty)$ is $\frac{1}{2}$-resolvent convex on $C$, then for any $x_{i} \in C, w_{i} \geq 0, i \in\{1, \ldots, n\}, n \geq 2$ with $W_{n}:=\sum_{i=1}^{n} w_{i}>0$ we have

$$
f\left(\frac{1}{W_{n}} \sum_{i=1}^{n} w_{i} x_{i}\right) \leq W_{n} \sum_{i=1}^{n} \frac{1}{2 W_{n}-w_{i}} f\left(x_{i}\right) .
$$

If the function $f: C \subseteq X \rightarrow[0, \infty)$ is $r$-exponential convex with $r$ fixed in $(0, \infty)$, then for any $x_{i} \in C, w_{i} \geq 0, i \in\{1, \ldots, n\}, n \geq 2$ with $W_{n}:=$ $\sum_{i=1}^{n} w_{i}>0$ we have

$$
f\left(\frac{1}{W_{n}} \sum_{i=1}^{n} w_{i} x_{i}\right) \leq \sum_{i=1}^{n} \exp \left[-r\left(1-\frac{w_{i}}{W_{n}}\right)\right] f\left(x_{i}\right) .
$$

\section{Some Related Functionals}

Let us fix $K \in \mathcal{P}_{f}(\mathbb{N})$ (the class of finite parts of $\mathbb{N}$ ) and $x_{i} \in C(i \in K)$. Now consider the functional $J_{K}: S_{+}(K) \rightarrow \mathbb{R}$ given by

$$
J_{K}(\mathbf{p}):=h\left(P_{K}\right) f\left(\frac{1}{P_{K}} \sum_{i \in K} p_{i} x_{i}\right) \geq 0
$$


where $S_{+}(K):=\left\{\mathbf{p}=\left(p_{i}\right)_{i \in I} \mid p_{i} \geq 0, i \in K\right.$ and $\left.P_{K}>0\right\}$ with $h:(0, \infty) \rightarrow$ $(0, \infty)$ and $f$ is nonnegative on $C$.

Theorem 4. Let $h:(0, \infty) \rightarrow(0, \infty)$ be a supermultiplicative (submultiplicative) function on $J$. If the function $f: C \subseteq X \rightarrow[0, \infty)$ is h-convex (h-concave) on the convex subset $C$ of the linear space $X$, then for any $\mathbf{p}, \mathbf{q} \in S_{+}(K)$ we have

$$
J_{K}(\mathbf{p}+\mathbf{q}) \leq(\geq) J_{K}(\mathbf{p})+J_{K}(\mathbf{q}),
$$

i.e., $J_{K}$ is a subadditive (superadditive) functional on $S_{+}(K)$.

Proof. If the function $f: C \subseteq X \rightarrow[0, \infty)$ is $h$-convex, then we have for any $\mathbf{p}, \mathbf{q} \in S_{+}(K)$

$$
\begin{aligned}
& J_{K}(\mathbf{p}+\mathbf{q}) \\
& =h\left(P_{K}+Q_{K}\right) f\left(\frac{1}{P_{K}+P_{K}} \sum_{i \in K}\left(p_{i}+q_{i}\right) x_{i}\right) \\
& =h\left(P_{K}+Q_{K}\right) f\left(\frac{P_{K} \cdot \frac{1}{P_{K}} \sum_{i \in K} p_{i} x_{i}+Q_{K} \cdot \frac{1}{Q_{K}} \sum_{i \in K} q_{i} x_{i}}{P_{K}+P_{K}}\right) \\
& \leq h\left(P_{K}+Q_{K}\right)\left[h\left(\frac{P_{K}}{P_{K}+P_{K}}\right) f\left(\frac{1}{P_{K}} \sum_{i \in K} p_{i} x_{i}\right)\right. \\
& \left.+h\left(\frac{Q_{K}}{P_{K}+P_{K}}\right) f\left(\frac{1}{Q_{K}} \sum_{i \in K} q_{i} x_{i}\right)\right] \\
& :=A .
\end{aligned}
$$

Since $h$ is supermultiplicative, then

$$
h\left(P_{K}+Q_{K}\right) h\left(\frac{P_{K}}{P_{K}+P_{K}}\right) \leq h\left(P_{K}\right)
$$

and

$$
h\left(P_{K}+Q_{K}\right) h\left(\frac{Q_{K}}{P_{K}+P_{K}}\right) \leq h\left(Q_{K}\right)
$$

which imply that

$$
\begin{aligned}
A & \leq h\left(P_{K}\right) f\left(\frac{1}{P_{K}} \sum_{i \in K} p_{i} x_{i}\right)+h\left(Q_{K}\right) f\left(\frac{1}{Q_{K}} \sum_{i \in K} q_{i} x_{i}\right) \\
& =J_{K}(\mathbf{p})+J_{K}(\mathbf{q}) .
\end{aligned}
$$

Making use of (3.3) and (3.4) we deduce the desired result (3.2).

The case when $h$ is submultiplicative and $f: C \subseteq X \rightarrow[0, \infty)$ is $h$-concave goes likewise and the details are omitted. 
Corollary 5. Let $h:(0, \infty) \rightarrow(0, \infty)$ be a submultiplicative function on $J$. If the function $f: C \subseteq X \rightarrow[0, \infty)$ is $h$-concave on the convex subset $C$ of the linear space $X$, then for any $\mathbf{p}, \mathbf{q} \in S_{+}(K)$ with $\mathbf{p} \geq \mathbf{q}$, i.e. $p_{i} \geq q_{i}$ for any $i \in K$, we have

$$
J_{K}(\mathbf{p}) \geq J_{K}(\mathbf{q}) \geq 0
$$

i.e., $J_{K}$ is monotonic nondecreasing on $S_{+}(K)$.

The proof is obvious from (3.2) on noticing that

$$
J_{K}(\mathbf{p})=J_{K}(\mathbf{p}-\mathbf{q}+\mathbf{q}) \geq J_{K}(\mathbf{p}-\mathbf{q})+J_{K}(\mathbf{q}) \geq J_{K}(\mathbf{q}) .
$$

We also have:

Corollary 6. Let $h:(0, \infty) \rightarrow(0, \infty)$ be a submultiplicative function on $J$. If the function $f: C \subseteq X \rightarrow[0, \infty)$ is $h$-concave on the convex subset $C$ of the linear space $X$, then for any $\mathbf{p}, \mathbf{q} \in S_{+}(K)$ with $M \mathbf{p} \geq \mathbf{q} \geq m \mathbf{p}$, for some $M>m>0$, we have

$$
\frac{h\left(M P_{K}\right)}{h\left(P_{K}\right)} J_{K}(\mathbf{p}) \geq J_{K}(\mathbf{q}) \geq \frac{h\left(m P_{K}\right)}{h\left(P_{K}\right)} J_{K}(\mathbf{p}) .
$$

Proof. From the inequality (3.5) we have

$$
J_{K}(M \mathbf{p}) \geq J_{K}(\mathbf{q}) .
$$

However

$$
\begin{aligned}
J_{K}(M \mathbf{p}) & =h\left(M P_{K}\right) f\left(\frac{1}{M P_{K}} \sum_{i \in K} M p_{i} x_{i}\right) \\
& =h\left(M P_{K}\right) f\left(\frac{1}{P_{K}} \sum_{i \in K} p_{i} x_{i}\right)=\frac{h\left(M P_{K}\right)}{h\left(P_{K}\right)} J_{K}(\mathbf{p}),
\end{aligned}
$$

which proves the first inequality in (3.6).

The second inequality can be proved similarly and the details are omitted.

Further, consider the functional $L_{K}: S_{+}(K) \rightarrow \mathbb{R}$ given by

$$
L_{K}(\mathbf{p}):=h\left(P_{K}\right) \sum_{i \in K} h\left(\frac{p_{i}}{P_{K}}\right) f\left(x_{i}\right) \geq 0,
$$

where $S_{+}(K):=\left\{\mathbf{p}=\left(p_{i}\right)_{i \in I} \mid p_{i} \geq 0, i \in K\right.$ and $\left.P_{K}>0\right\}$ with $h:(0, \infty) \rightarrow$ $(0, \infty)$ and $f$ is nonnegative on $C$.

Theorem 5. Let $h:(0, \infty) \rightarrow(0, \infty)$ and $f: C \subseteq X \rightarrow[0, \infty)$. If $h$ is convex (concave) on $(0, \infty)$ and $g:(0, \infty) \rightarrow(0, \infty)$ defined by $g(t)=\frac{h(t)}{t}$ is decreasing (increasing), then for any $\mathbf{p}, \mathbf{q} \in S_{+}(K)$ we have

$$
L_{K}(\mathbf{p}+\mathbf{q}) \leq(\geq) L_{K}(\mathbf{p})+L_{K}(\mathbf{q}) .
$$


Proof. If $h$ is convex on $(0, \infty)$, then we have for any $\mathbf{p}, \mathbf{q} \in S_{+}(K)$

$$
\begin{aligned}
L_{K}(\mathbf{p}+\mathbf{q}) & =h\left(P_{K}+Q_{K}\right) \sum_{i \in K} h\left(\frac{p_{i}+q_{i}}{P_{K}+Q_{K}}\right) f\left(x_{i}\right) \\
& =h\left(P_{K}+Q_{K}\right) \sum_{i \in K} h\left(\frac{P_{K} \frac{p_{i}}{P_{K}}+Q_{K} \frac{q_{i}}{Q_{K}}}{P_{K}+Q_{K}}\right) f\left(x_{i}\right) \\
\leq & h\left(P_{K}+Q_{K}\right) \times \sum_{i \in K}\left[\frac{P_{K}}{P_{K}+Q_{K}} h\left(\frac{p_{i}}{P_{K}}\right)\right. \\
& \left.+\frac{Q_{K}}{P_{K}+Q_{K}} h\left(\frac{q_{i}}{Q_{K}}\right)\right] f\left(x_{i}\right) \\
& =\frac{h\left(P_{K}+Q_{K}\right) P_{K}}{P_{K}+Q_{K}} \sum_{i \in K} h\left(\frac{p_{i}}{P_{K}}\right) f\left(x_{i}\right) \\
& +\frac{h\left(P_{K}+Q_{K}\right) Q_{K}}{P_{K}+Q_{K}} \sum_{i \in K} h\left(\frac{q_{i}}{Q_{K}}\right) f\left(x_{i}\right) \\
& :=B .
\end{aligned}
$$

Since $g(t)=\frac{h(t)}{t}$ is decreasing, then

$$
\frac{h\left(P_{K}+Q_{K}\right)}{P_{K}+Q_{K}} \leq \frac{h\left(P_{K}\right)}{P_{K}}
$$

and

Therefore

$$
\frac{h\left(P_{K}+Q_{K}\right)}{P_{K}+Q_{K}} \leq \frac{h\left(Q_{K}\right)}{Q_{K}}
$$

$$
\begin{aligned}
B & \leq h\left(P_{K}\right) \sum_{i \in K} h\left(\frac{p_{i}}{P_{K}}\right) f\left(x_{i}\right)+h\left(Q_{K}\right) \sum_{i \in K} h\left(\frac{q_{i}}{Q_{K}}\right) f\left(x_{i}\right) \\
& =L_{K}(\mathbf{p})+L_{K}(\mathbf{q}) .
\end{aligned}
$$

Making use of (3.9) and (3.10) we deduce the desired result (3.8).

The case when $h$ is concave and $g$ is increasing goes likewise and the details are omitted.

Corollary 7. Let $h:(0, \infty) \rightarrow(0, \infty)$ and $f: C \subseteq X \rightarrow[0, \infty)$. If $h$ is concave on $(0, \infty)$ and $g:(0, \infty) \rightarrow(0, \infty)$ defined by $g(t)=\frac{h(t)}{t}$ is increasing, then for any $\mathbf{p}, \mathbf{q} \in S_{+}(K)$ with $\mathbf{p} \geq \mathbf{q}$ we have

$$
L_{K}(\mathbf{p}) \geq L_{K}(\mathbf{q}) \geq 0 .
$$

Also, for any $\mathbf{p}, \mathbf{q} \in S_{+}(K)$ with $M \mathbf{p} \geq \mathbf{q} \geq m \mathbf{p}$, for some $M>m>0$, we have

$$
\frac{h\left(M P_{K}\right)}{h\left(P_{K}\right)} L_{K}(\mathbf{p}) \geq L_{K}(\mathbf{q}) \geq \frac{h\left(m P_{K}\right)}{h\left(P_{K}\right)} L_{K}(\mathbf{p}) .
$$


We define the difference functional

$$
\begin{aligned}
S_{K}(\mathbf{p}) & :=L_{K}(\mathbf{p})-J_{K}(\mathbf{p}) \\
& =h\left(P_{K}\right)\left[\sum_{i \in K} h\left(\frac{p_{i}}{P_{K}}\right) f\left(x_{i}\right)-f\left(\frac{1}{P_{K}} \sum_{i \in K} p_{i} x_{i}\right)\right] .
\end{aligned}
$$

We observe that, if $h$ is supermultiplicative and $f: C \subseteq X \rightarrow[0, \infty)$ is $h$-convex, then by Jensen's type inequality (1.7) we have

$$
S_{K}(\mathbf{p}) \geq 0 \text { for any } \mathbf{p} \in S_{+}(K) .
$$

Proposition 1. Let $h:(0, \infty) \rightarrow(0, \infty)$ be supermultiplicative and $f:$ $C \subseteq X \rightarrow[0, \infty)$ a h-convex function on $C$. If $h$ is concave on $(0, \infty)$ and $g:(0, \infty) \rightarrow(0, \infty)$ defined by $g(t)=\frac{h(t)}{t}$ is increasing, then for any $\mathbf{p}, \mathbf{q} \in S_{+}(K)$

$$
S_{K}(\mathbf{p}+\mathbf{q}) \geq S_{K}(\mathbf{p})+S_{K}(\mathbf{q}) \geq 0 .
$$

If $\mathbf{p}, \mathbf{q} \in S_{+}(K)$ with $\mathbf{p} \geq \mathbf{q}$, then we have

$$
S_{K}(\mathbf{p}) \geq S_{K}(\mathbf{q}) \geq 0 .
$$

Also, for any $\mathbf{p}, \mathbf{q} \in S_{+}(K)$ with $M \mathbf{p} \geq \mathbf{q} \geq m \mathbf{p}$, for some $M>m>0$, we have

$$
\frac{h\left(M P_{K}\right)}{h\left(P_{K}\right)} S_{K}(\mathbf{p}) \geq S_{K}(\mathbf{q}) \geq \frac{h\left(m P_{K}\right)}{h\left(P_{K}\right)} S_{K}(\mathbf{p}) .
$$

The proof follows by Theorem 4 and Theorem 5 and we omit the details. If we take $h(t)=t$, i.e. in the case of convex functions we obtain from Proposition 1 the superadditivity and monotonicity properties of the functional

$$
J e_{K}(\mathbf{p}):=\sum_{i \in K} p_{i} f\left(x_{i}\right)-P_{K} f\left(\frac{1}{P_{K}} \sum_{i \in K} p_{i} x_{i}\right)
$$

established in ( [32]).

From (3.15) we get

$$
M J e_{K}(\mathbf{p}) \geq J e_{K}(\mathbf{q}) \geq m J e_{K}(\mathbf{p})
$$

that has been obtained in [24].

\section{REFERENCES}

[1] M. Alomari and M. Darus, The Hadamard's inequality for s-convex function. Int. J. Math. Anal. (Ruse) 2 (2008), no. 13-16, 639-646.

[2] M. Alomari and M. Darus, Hadamard-type inequalities for s-convex functions. Int. Math. Forum 3 (2008), no. 37-40, 1965-1975.

[3] G. A. Anastassiou, Univariate Ostrowski inequalities, revisited. Monatsh. Math., 135 (2002), no. 3, 175-189. 
[4] N. S. Barnett, P. Cerone, S. S. Dragomir, M. R. Pinheiro,and A. Sofo, Ostrowski type inequalities for functions whose modulus of the derivatives are convex and applications. Inequality Theory and Applications, Vol. 2 (Chinju/Masan, 2001), 19-32, Nova Sci. Publ., Hauppauge, NY, 2003. Preprint: RGMIA Res. Rep. Coll. 5 (2002), No. 2, Art. 1 [Online http://rgmia.org/papers/v5n2/Paperwapp2q.pdf].

[5] E. F. Beckenbach, Convex functions, Bull. Amer. Math. Soc. 54(1948), 439-460.

[6] M. Bombardelli and S. Varošanec, Properties of h-convex functions related to the Hermite-Hadamard-Fejér inequalities. Comput. Math. Appl. 58 (2009), no. 9, 18691877.

[7] W. W. Breckner, Stetigkeitsaussagen für eine Klasse verallgemeinerter konvexer Funktionen in topologischen linearen Räumen. (German) Publ. Inst. Math. (Beograd) (N.S.) 23(37) (1978), 13-20.

[8] W. W. Breckner and G. Orbán, Continuity properties of rationally s-convex mappings with values in an ordered topological linear space. Universitatea "Babeş-Bolyai", Facultatea de Matematica, Cluj-Napoca, 1978. viii+92 pp.

[9] P. Cerone and S. S. Dragomir, Midpoint-type rules from an inequalities point of view, Ed. G. A. Anastassiou, Handbook of Analytic-Computational Methods in Applied Mathematics, CRC Press, New York. 135-200.

[10] P. Cerone and S. S. Dragomir, New bounds for the three-point rule involving the Riemann-Stieltjes integrals, in Advances in Statistics Combinatorics and Related Areas, C. Gulati, et al. (Eds.), World Science Publishing, 2002, 53-62.

[11] P. Cerone, S. S. Dragomir and J. Roumeliotis, Some Ostrowski type inequalities for $n$-time differentiable mappings and applications, Demonstratio Mathematica, 32(2) (1999), 697-712.

[12] G. Cristescu, Hadamard type inequalities for convolution of $h$-convex functions. Ann. Tiberiu Popoviciu Semin. Funct. Equ. Approx. Convexity 8 (2010), 3-11.

[13] S. S. Dragomir, Ostrowski's inequality for monotonous mappings and applications, J. KSIAM, 3(1) (1999), 127-135.

[14] S. S. Dragomir, The Ostrowski's integral inequality for Lipschitzian mappings and applications, Comp. Math. Appl., 38 (1999), 33-37.

[15] S. S. Dragomir, On the Ostrowski's inequality for Riemann-Stieltjes integral, Korean J. Appl. Math., 7 (2000), 477-485.

[16] S. S. Dragomir, On the Ostrowski's inequality for mappings of bounded variation and applications, Math. Ineq. ES Appl., 4(1) (2001), 33-40.

[17] S. S. Dragomir, On the Ostrowski inequality for Riemann-Stieltjes integral $\int_{a}^{b} f(t) d u(t)$ where $f$ is of Hölder type and $u$ is of bounded variation and applications, J. KSIAM, 5(1) (2001), 35-45.

[18] S. S. Dragomir, Ostrowski type inequalities for isotonic linear functionals, J. Inequal. Pure 85 Appl. Math., 3(5) (2002), Art. 68.

[19] S. S. Dragomir, An inequality improving the first Hermite-Hadamard inequality for convex functions defined on linear spaces and applications for semi-inner products. J. Inequal. Pure Appl. Math. 3 (2002), no. 2, Article 31, 8 pp.

[20] S. S. Dragomir, An inequality improving the first Hermite-Hadamard inequality for convex functions defined on linear spaces and applications for semi-inner products, J. Inequal. Pure Appl. Math. 3 (2002), No. 2, Article 31.

[21] S. S. Dragomir, An inequality improving the second Hermite-Hadamard inequality for convex functions defined on linear spaces and applications for semi-inner products, J. Inequal. Pure Appl. Math. 3 (2002), No.3, Article 35. 
[22] S. S. Dragomir, An Ostrowski like inequality for convex functions and applications, Revista Math. Complutense, 16(2) (2003), 373-382.

[23] S. S. Dragomir, Operator Inequalities of Ostrowski and Trapezoidal Type. Springer Briefs in Mathematics. Springer, New York, 2012. x+112 pp. ISBN: 978-1-4614-17781

[24] S. S. Dragomir, Bounds for the normalised Jensen functional, Bull. Austral. Math. Soc. 74 (2006), pp. 471-478.

[25] S. S. Dragomir, P. Cerone, J. Roumeliotis and S. Wang, A weighted version of Ostrowski inequality for mappings of Hölder type and applications in numerical analysis, Bull. Math. Soc. Sci. Math. Romanie, 42(90) (4) (1999), 301-314.

[26] S.S. Dragomir and S. Fitzpatrick, The Hadamard inequalities for s-convex functions in the second sense. Demonstratio Math. 32 (1999), no. 4, 687-696.

[27] S.S. Dragomir and S. Fitzpatrick, The Jensen inequality for s-Breckner convex functions in linear spaces. Demonstratio Math. 33 (2000), no. 1, 43-49.

[28] S. S. Dragomir and B. Mond, On Hadamard's inequality for a class of functions of Godunova and Levin. Indian J. Math. 39 (1997), no. 1, 1-9.

[29] S. S. Dragomir and C. E. M. Pearce, On Jensen's inequality for a class of functions of Godunova and Levin. Period. Math. Hungar. 33 (1996), no. 2, 93-100.

[30] S. S. Dragomir and C. E. M. Pearce, Quasi-convex functions and Hadamard's inequality, Bull. Austral. Math. Soc. 57 (1998), 377-385.

[31] S. S. Dragomir, J. Pečarić and L. Persson, Some inequalities of Hadamard type. Soochow J. Math. 21 (1995), no. 3, 335-341.

[32] S. S. Dragomir, J. Pečarić and L. Persson, Properties of some functionals related to Jensen's inequality, Acta Math. Hungarica, 70 (1996), 129-143.

[33] S. S. Dragomir and Th. M. Rassias (Eds), Ostrowski Type Inequalities and Applications in Numerical Integration, Kluwer Academic Publisher, 2002.

[34] S. S. Dragomir and S. Wang, A new inequality of Ostrowski's type in $L_{1}$-norm and applications to some special means and to some numerical quadrature rules, Tamkang J. of Math., 28 (1997), 239-244.

[35] S. S. Dragomir and S. Wang, Applications of Ostrowski's inequality to the estimation of error bounds for some special means and some numerical quadrature rules, Appl. Math. Lett., 11 (1998), 105-109.

[36] S. S. Dragomir and S. Wang, A new inequality of Ostrowski's type in $L_{p}-$ norm and applications to some special means and to some numerical quadrature rules, Indian J. of Math., 40(3) (1998), 245-304.

[37] A. El Farissi, Simple proof and refinement of Hermite-Hadamard inequality, J. Math. Ineq. 4 (2010), No. 3, 365-369.

[38] E. K. Godunova and V. I. Levin, Inequalities for functions of a broad class that contains convex, monotone and some other forms of functions. (Russian) Numerical mathematics and mathematical physics (Russian), 138-142, 166, Moskov. Gos. Ped. Inst., Moscow, 1985

[39] H. Hudzik and L. Maligranda, Some remarks on s-convex functions. Aequationes Math. 48 (1994), no. 1, 100-111.

[40] E. Kikianty and S. S. Dragomir, Hermite-Hadamard's inequality and the p-HH-norm on the Cartesian product of two copies of a normed space, Math. Inequal. Appl. (in press)

[41] U. S. Kirmaci, M. Klaričić Bakula, M. E Özdemir and J. Pečarić, Hadamard-type inequalities for s-convex functions. Appl. Math. Comput. 193 (2007), no. 1, 26-35. 
[42] M. A. Latif, On some inequalities for h-convex functions. Int. J. Math. Anal. (Ruse) 4 (2010), no. 29-32, 1473-1482.

[43] D. S. Mitrinović and I. B. Lacković, Hermite and convexity, Aequationes Math. 28 (1985), 229-232.

[44] D. S. Mitrinović and J. E. Pečarić, Note on a class of functions of Godunova and Levin. C. R. Math. Rep. Acad. Sci. Canada 12 (1990), no. 1, 33-36.

[45] C. E. M. Pearce and A. M. Rubinov, P-functions, quasi-convex functions, and Hadamard-type inequalities. J. Math. Anal. Appl. 240 (1999), no. 1, 92-104.

[46] J. E. Pečarić and S. S. Dragomir, On an inequality of Godunova-Levin and some refinements of Jensen integral inequality. Itinerant Seminar on Functional Equations, Approximation and Convexity (Cluj-Napoca, 1989), 263-268, Preprint, 89-6, Univ. "Babeş-Bolyai", Cluj-Napoca, 1989.

[47] J. Pečarić and S. S. Dragomir, A generalization of Hadamard's inequality for isotonic linear functionals, Radovi Mat. (Sarajevo) 7 (1991), 103-107.

[48] M. Radulescu, S. Radulescu and P. Alexandrescu, On the Godunova-Levin-Schur class of functions. Math. Inequal. Appl. 12 (2009), no. 4, 853-862.

[49] M. Z. Sarikaya, A. Saglam, and H. Yildirim, On some Hadamard-type inequalities for h-convex functions. J. Math. Inequal. 2 (2008), no. 3, 335-341.

[50] E. Set, M. E. Özdemir and M. Z. Sarıkaya, New inequalities of Ostrowski's type for s-convex functions in the second sense with applications. Facta Univ. Ser. Math. Inform. 27 (2012), no. 1, 67-82.

[51] M. Z. Sarikaya, E. Set and M. E. Özdemir, On some new inequalities of Hadamard type involving h-convex functions. Acta Math. Univ. Comenian. (N.S.) 79 (2010), no. $2,265-272$.

[52] M. Tunç, Ostrowski-type inequalities via h-convex functions with applications to special means. J. Inequal. Appl. 2013, 2013:326.

[53] S. Varošanec, On h-convexity. J. Math. Anal. Appl. 326 (2007), no. 1, 303-311.

Address:

Professor Silvestru Sever Dragomir

Mathematics, College of Engineering \& Science

Victoria University, PO Box 14428

Melbourne City, MC 8001

Australia

Secondary address:

School of Computational \& Applied Mathematics UNIVERSity OF THE WitwatersRAND

Private Bag 3

JOHANNESBURG 2050

South AFricA

E-mail address: sever.dragomir@vu.edu.au

$U R L:$ http://rgmia.org/dragomir 